\title{
Rappresentare l'architettura fortificata per narrare e valorizzare il territorio della frontiera alessandrina
}

Representing fortified architecture to narrate and enhance the Alexandrian territory border

\author{
Anna Marotta ${ }^{\text {a }}$, Vincenzo Cirillo ${ }^{b}$, Claudio Rabino ${ }^{c}$, Ornella Zerlenga ${ }^{d}$ \\ a Politecnico di Torino, Turin, Italy, anna.marotta@polito.it \\ b Università degli Studi della Campania “Luigi Vanvitelli”, Aversa, Italy, vincenzo.cirillo@unicampania.it \\ ${ }^{\mathrm{c}}$ Politecnico di Torino, Turin, Italy, claudio.rabino@polito.it \\ 'Università degli Studi della Campania “Luigi Vanvitelli”, Aversa, Italy, ornella.zerlenga@unicampania.it
}

\begin{abstract}
The Piemonte cultural territory is also characterized by the significant presence of complex defensive systems, grouped by types and orographic configuration. Specifically,this paper will address asystematic and unitary re-reading of the territory of the Alessandria area, which includes: the sixteenth-century Cittadella di Casale, of the Gonzagas; the Citadel of Alexandria (1732), by Giulio Ignazio Bertola; Valenza and its fortifications (from the "Spanish" period with interventions by Gaspare Beretta, also interested in the fort / Castello Tortona); that of Gavi (restored "in the modern" by Gaspare Maculano known as Fiorenzuola, the friar who condemned Galileo). Following thirty years international research, the objectives of the work are twofold: the first aims to physically connect in this system a "network" of thematically dedicated tourist itineraries, through documentary paths that reconstruct historical events, construction phases and transformation, (in similitude with the ECCOFORT project, in the province of Verona). Following other works, by the authors already dedicated to the Alexandrian, through the advanced 3D modeling (like the one carried out in the reconstruction of the fort of Gavi) the second objective of the present contribution will follow the same restoring and reconstructive procedure. Through digitalized anastylosis, even dynamized, the Castle of Tortona will be rebuilt on the basis -among other things- of the conspicuous documentation (dated 1799, the year of the Napoleonic siege, followed by demolition, in 1801) found at the Osterreichisches Staatsarchiv, Kriegsarchiv of Vienna. The critical reconstruction is now made possible thanks to the precious wealth of knowledge, matured over the decades by Marotta, Zerlenga, Abello: with (indexed) data, including metrics, graphics and visual returns. Drawing between real and virtual, it will be possible to reconnect individual episodes, comparable and critically selected information, by means of congruently connected interventions, both physically and digitally. Finally, in a project of knowledge, conservation and valorisation, the "local" dimension dialogues and integrates with the European dimension.
\end{abstract}

Keywords: Defense territories in Europe, Alexandrian borders, fortifications, defensive typologies, 3D modeling.

\section{Introduzione}

Per comprendere perché il territorio alessandrino (Fig. 1) si sia trasformato in terra di confine con una forte valenza strategico-militare bisogna partire dal secolo XIV, quando l'area entrò a far 
parte del Ducato Visconteo di Milano, grazie a un diploma del 1395 di Venceslao di Lussemburgo, imperatore del Sacro Romano Impero. Un documento (del 1396) riporta i centri più importanti del territorio, tra cui Alessandria e Tortona. Nel tempo le frontiere del Ducato si modificarono in modo rilevante fino a coinvolgere, anche se a volte per pochi anni, nella loro massima espansione a ovest oltre all'area alessandrina, l'astigiano fino al monregalese, mentre ad est giungevano fino a Belluno e a sud inglobavano $\mathrm{i}$ territori di Pisa, Siena, Perugia e Assisi. Alle alterne e complesse sorti del Ducato di Milano il territorio alessandrino legò la sua storia di regione di frontiera, posta a cavaliere tra questo Ducato ad est, la contea di Savoia a nord e la Repubblica di Genova a sud, fino all'epopea napoleonica, che annullò stati e confini tra XVIII e il XIX secolo.

\section{L'Italia settentrionale e le mire franco- spagnole}

Con la morte di Filippo Maria, nel 1447, terminò il dominio visconteo: nacque a Milano una nuova forma di governo, della Repubblica Ambrosiana, la cui difesa fu affidata nel 1450 ad un valente uomo d'armi, uno Sforza, Ludovico detto il Moro: con lui termina la Repubblica, e nasce il nuovo Ducato del suo casato. Fu lui ad incoraggiare, nel 1494, la calata in Italia del re di Francia Carlo VIII per rivendicare il trono di Napoli, in mano agli Aragonesi, sconvolgendo inesorabilmente gli equilibri tra i vari stati italiani e dando inizio a quelle che passeranno alla storia come le Guerre d'Italia (1494-1559). Nel 1495 Carlo VIII fu scacciato dalla Penisola, ma il danno era fatto. Dopo un ventennio di alterne vicende, nel 1515 Francesco I di Valois ristabilì la corona francese sul Ducato, fino a quando Carlo $\mathrm{V}$, Re di Spagna e Imperatore del Sacro Romano Impero, non rimise sul trono del Ducato uno Sforza, Francesco II, che governò fino al 1535. Fu così che gli Spagnoli iniziarono a guardare, con interesse sempre crescente, il Ducato di Milano. Con la morte senza eredi di Francesco Maria, Carlo V decise di annettere il Ducato di Milano ai suoi domini, per evitare che passasse al suo nemico Francesco I, conferendo nel 1540, il titolo di duca a suo figlio Filippo.

\section{La pace di Cateau Cambrésis}

$\mathrm{Nel}$ frattempo, l'Alessandrino era sotto la dominazione francese, come buona parte del Piemonte. Per vedere il concretizzarsi di una nuova realtà politico-militare in tali territori, bisognerà attendere il 1557, e la battaglia di San Quintino, a conclusione delle cosiddette "Guerre italiane". La seguente pace di Cateau Cambrésis (1559), vedrà Emanuele Filiberto di Savoia premiato con la restituzione del Piemonte e della Savoia da parte della Francia. Se con questo accordo inizia per l'Italia un lungo periodo di asservimento agli Spagnoli, tornati in possesso del Regno di Napoli, della Sicilia, della Sardegna, della Lombardia e dello Stato dei Presidi (in Toscana), per la terra alessandrina inizia a delinearsi quel territorio di frontiera che contraddistinguerà e influenzerà la vita politico-militare dell'area per un lunghissimo periodo. Il Piemonte si sta concretizzando nella sua conformazione geografico-politica che noi ancora riconosciamo e la frontiera con il Ducato di Milano sotto dominazione spagnola si colloca a pochi chilometri dal confine territoriale di Alessandria. A nord i Gonzaga fortificati nella Cittadella di Casale Monferrato (Marotta, 1990a), ad est gli Spagnoli, a sud i Genovesi, a ovest i Francesi. Caso unico al tempo in Europa, la cittadella esagonale di Casale Monferrato è quella che le ha dato il titolo di "città piazzaforte". Progettata e governata in tempi successivi da Stati diversi, dai Paleologi, ai Gonzaga, agli Spagnoli di Milano, e infine ai Francesi di Luigi XIV, la costruzione inizia nel 1589-1590, terminando nel 1595. Progettata dal friulano Germanico Savorgnan, chiamato da Vincenzo I Gonzaga, sono noti -fra l'altro- contributi di Bernardino Faciotto. Successivamente, subì due importanti assedi -entrambi senza effetto- da parte dell'esercito ispanico: il primo (1628) fu compiuto dall'Armata spagnola comandata da Don Gonsalvo de Cordova; il secondo attacco, del 1630, dalla stessa l'Armata agli ordini questa volta del marchese genovese Ambrogio Spinola. Il duca di Mantova, Ferdinando Carlo (regnante dal 1665 al 1707), in lotta con gli Spagnoli, intraprese trattative segrete con il re di Francia Luigi XIV per la cessione del controllo di Casale e della sua Cittadella, resa che doveva tuttavia risultare quale esito di un'azione di forza. In ba- 
se all'accordo, Luigi XIV comandò il finto assedio nel dicembre 1681, quando la guarnigione del generale francese Catinat assunse il comando del presidio casalese.

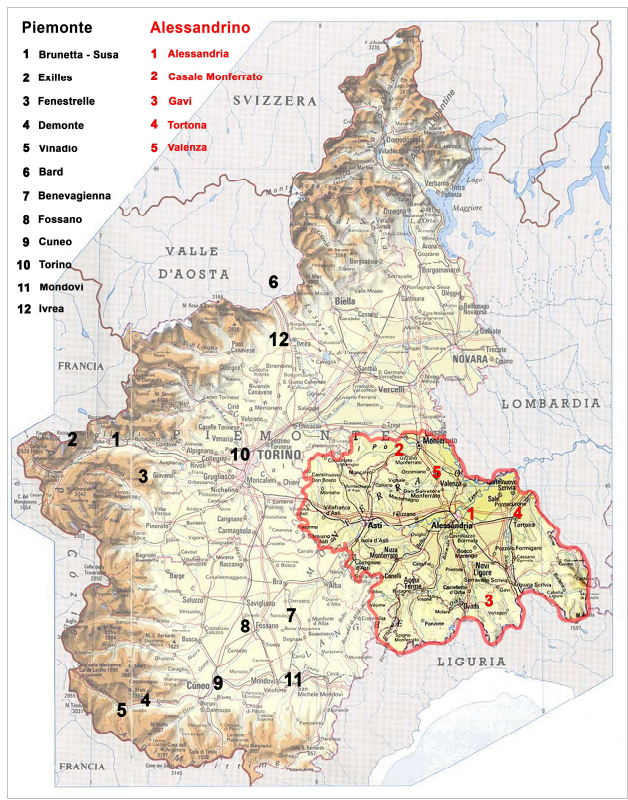

Fig. 1. Il territorio alessandrino con l'identificazione dei principali luoghi fortificati di carattere strategicomilitare.

L'effettiva dismissione, da parte del marchese di Crenau, avvenne nel 1695. Infine, tra il 1850 ed il 1859, nelle Guerre di Indipendenza italiana nell'area della preesistente fortezza gonzaghesca- si realizzò una "corona" bastionata, nel sistema del "campo trincerato" di Casale, determinante per la linea difensiva che La Marmora aveva organizzato da Torino al LombardoVeneto.

A riprova dell'importanza di tale frontiera, specie verso est, basti ricordare che a pochi passi da Alessandria la fortezza di Valenza (Fig. 2) per lungo tempo francese, venne assegnata dopo il trattato di Cateau Cambrésis agli Spagnoli del Ducato di Milano. Ma nel 1635, durante la Guerra dei Trent'anni, la fortezza di Valenza fu messa per 60 giorni sotto assedio dai Francesi e dai Piemontesi savoiardi e resistette anche al secondo assedio franco-piemontese del 1641, per capitolare dopo 70 giorni di assedio alle truppe fran- cesi, piemontesi e modenesi nel 1556. La guerra di frontiera continuerà inesorabilmente fino all'assedio del 1696, durante la cosiddetta Guerra della Lega d'Austria contro Luigi XIV, e anche in quell'occasione Francesi e Sabaudi non riuscirono ad espugnarla. Fu poi definitivamente conquistata nel 1707, durante la Guerra di Successione spagnola dal Duca Vittorio Amedeo II di Savoia, possesso confermato dal successivo trattato di Utrecht del 1713. Ancora a seguito dello stesso conflitto, e dello stesso Trattato, l'assegnazione di Alessandria ai Savoia diventa definitiva e costituisce il primo importante passo dell'espansione della Savoia sul versante italiano. Nel 1728 Vittorio Amedeo II, dopo la sua ascesa al trono, ordina a Ignazio Bertola di Roveda "maestro di fortificazioni" della Casa Reale, e uno dei più importanti progettisti di fortificazioni del XVIII secolo, di progettare ed erigere una nuova cittadella ad Alessandria: una delle migliorie più vaste in Europa, progettata in forma di esagono regolare secondo teorie di scuola francese (Vauban) e olandese (Coehorn) (Figg. 3a e 5d) (Marotta, 1990b). Riprendendo le vicende dal XVII secolo, va annotato come, a rafforzare il ruolo strategico della frontiera alessandrina fu non solo la sua collocazione geografica, ma innanzitutto l'ambigua e ondivaga politica dei Duchi di Savoia che passavano in maniera disinvolta dalle file degli alleati spagnoli, a quelle momentaneamente "più sicure" dei Francesi, e viceversa. Nei pressi della frontiera alessandrina passava una strada di importanza fondamentale per la Spagna, perché collegava il porto di Savona e di Vado attraverso la Lombardia e la Valtellina e quindi i possedimenti spagnoli nel sud Europa con quelli nel nord e nelle Fiandre. Per tale ragione, la fortezza di Tortona, (Fig. 5b) (Mandracci, Marotta, 1995) posta a cavallo del cammino, assunse una importanza strategica tale da giustificare guerre e assedi per il suo possesso: viene sostituita e/o ampliata la vecchia cinta bastionata di epoca viscontea e sforzesca con una nuova cinta "alla moderna". Per questa piazzaforte, fortemente provata dagli assedi subiti nel conflitto Franco-Piemontese degli anni 1642/43, inizia il recupero da parte dei governatori spagnoli dello Stato di Milano nella seconda metà del Seicento. 


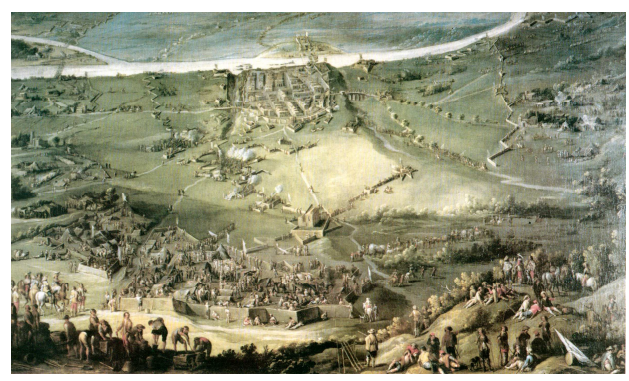

Fig. 2. Juan de la Corte, Valenza del Po, raffigurazione del soccorso portato da Colonna in occasione dell'assedio del 1635 (stralcio).

Responsabile dei profondi interventi di rinforzo è l'ingegnere capo dello Stato di Milano, Gaspare Beretta, che firmerà gli interventi sia sulla cinta bastionata che sul castello. Il 29 novembre 1706 la piazzaforte di Tortona si arrendé agli Austriaci nell'ambito della Guerra di Successione Spagnola. Sotto il dominio asburgico, negli anni Venti del Settecento, vengono riprogettati e ricostruiti o riparati parte delle cortine con l'aggiunta di nuovi rivellini e di baluardi, e il castello. E con questa nuova veste che Tortona, entrata nel 1738 a far parte dello Stato sardo, sarà nuovamente oggetto di assedio nel 1745 da parte dei Galli-Ispanici. Ritornata ai Savoia, la fortezza viene riprogettata dall'ingegnere Lorenzo Bernardino Pinto che ridisegna il castello, trasformandolo in un vero forte, con nuove ridotte, bastioni, controscarpe e caserme e con particolare attenzione agli accessi ufficiali del forte stesso (Fig. 4). Dopo l'assedio del 1799 ad opera delle truppe napoleoniche, a riprova dell'importanza assunta dalla piazzaforte, nel 1801, lo stesso Bonaparte decreta la totale distruzione della fortezza.

A questo punto appare indispensabile tornare indietro nel tempo, e ripartire dalla Pace di Cateau Cambresis, che non garantì del tutto la fase di relativa tranquillità -dal 1559 al 1620- che non fu esente da grossi eventi bellici, alcuni dei quali avvennero proprio nel territorio alessandrino, come la prima guerra del Monferrato tra gli Spagnoli e Carlo Emanuele I di Savoia nel 1610.

Il Ducato del Monferrato, con capitale Casale, al limitare nord della frontiera alessandrina, con la sua imponente Cittadella costruita sul finire del
XVI secolo (Fig. 5c), era collocata -come già accennato- in posizione strategicamente importante, in una ulteriore via di passaggio per connettere il Ducato di Milano con il porto di Genova, quindi con la Spagna. Il Ducato del Monferrato era controllato in via indiretta dagli Spagnoli attraverso i loro alleati, i Gonzaga di Mantova. Alla morte del duca Francesco IV Gonzaga -nel 1612- i Savoia rivendicarono il possesso del Ducato del Monferrato. Ma la Spagna era di tutt'altro avviso, per cui nel 1613 scoppiò il conflitto tra lo Stato sabaudo e gli Spagnoli di Milano: i territori piemontesi furono invasi dalle truppe spagnole, senza grandi risultati, tant'è che con il trattato di Asti del 1615, voluto dai Francesi (fortemente intenzionati a non restare inattivi) si pose fine alla prima fase del conflitto. In quegli anni, la complessa situazione fra Spagnoli, Francesi (e i Savoia) fà sì che le fortezze sulla frontiera alessandrina continuino a non avere pace. Nel 1627 muore il Duca Vincenzo II Gonzaga, senza lasciare eredi. La successione spettava ad un principe francese (Carlo I di GonzagaNevers) che, avendo il pieno appoggio del re di Francia Luigi XIII, avanzò i suoi diritti alla successione e si impossessò di Mantova il 17 gennaio del 1628. Gli Spagnoli gli opposero la candidatura di Ferrante II Gonzaga. I Francesi intervennero e come reazione si mossero i Piemontesi del duca di Savoia Carlo Emanuele I. I Sabaudi occupano alcune città ai confini nord e ovest del territorio alessandrino, Trino, Alba e Moncalvo, a loro volta le truppe spagnole (guidate Ambrogio Spinola) attaccarono la piazzaforte di Casale. Quando Luigi XIII terminò -vincendol'assedio di La Rochelle contro i protestanti ugonotti, scese velocemente in Italia, liberando la fortezza di Casale e occupando quella di Pinerolo. Gli Spagnoli reagirono assediando Mantova, e di conseguenza la piazzaforte di Casale. Alla fine, nel 1631, con la pace di Cherasco, tutti i contendenti trovarono un accordo, scambiandosi (quali pezzi di un gioco a scacchi) territori e città. Per ciò che qui interessa maggiormente, al duca Vittorio Amedeo di Savoia la Francia cedette molte terre del Monferrato, ma alla medesima nazione si dovette lasciare il possesso della fortezza di Pinerolo. Dopo un trentennio di catastrofi per la Lombardia, terminato intorno al 
1660, a inizio del Settecento, dopo la cosiddetta Guerra della Lega di Augusta -guerreggiata anche nel territorio piemontese, ma lontano dalle frontiere e dalle fortezze alessandrine- si affacciò al confine est un nuovo soggetto politicomilitare: l'Austria. Alla morte, senza eredi, di Carlo II di Spagna, scoppiò un lungo conflitto: la Guerra di Successione Spagnola (1701/1715) divampata in tutta Europa e coinvolse, ovviamente, anche la Lombardia. Alla fine, fu l'Austria nel 1706 ad affermarsi come nuovo dominus di questa regione, mentre venivano definitivamente legate ai domini sabaudi le città di Valenza e quella di Alessandria.

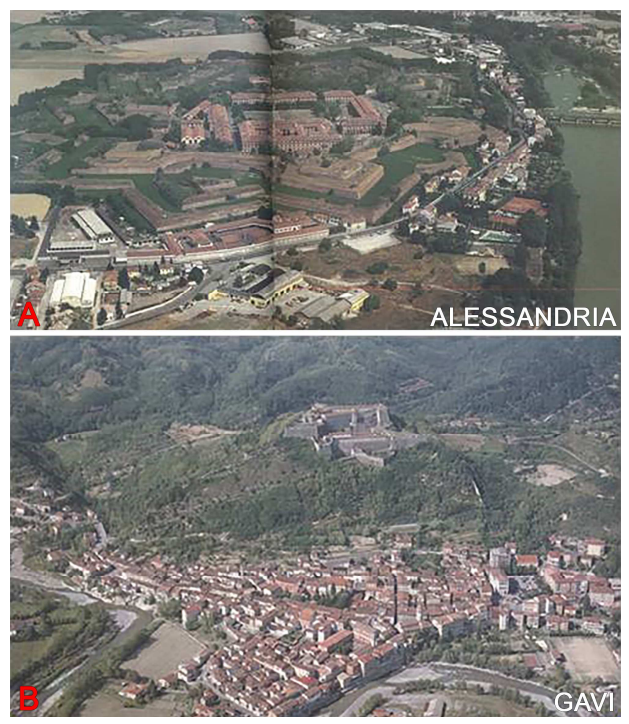

Fig. 3. Cittadella di Alessandria (a) e il Forte di Gavi (b) all'attualità con una vista dall'alto.

\section{I ridimensionamenti della frontiera nel ter- ritorio alessandrino con l'Austria in Lombar- dia}

Dopo un ventennio di pace, il trattato di Utrecht fu impugnato dall'imperatore austriaco Carlo VI e questa presa di posizione si trasformò presto in guerra tra la stessa Austria e i Franco-Piemontesi che invasero la Lombardia, dal 1733 al 1736. Due anni dopo i contendenti stipularono la pace di Vienna, con il ritorno degli Austriaci in Lombardia e la cessione allo Stato Sabaudo della città di Novara e di Tortona. Ancora una volta la frontiera alessandrina si vede modificata, accre- scendo il suo alto potere strategico: è quanto si confermerà con la Guerra di Successione austriaca e che interesserà molto da vicino i territori alessandrini. Il conflitto fu combattuto su un vastissimo territorio, anche in Italia dove le due coalizioni, la franco-spagnola e quella antifrancese con a capo l'Austria, si scontrarono in numerose battaglie. Nella prima fase della guerra la coalizione franco-spagnola dilagò in Piemonte e proprio nell'Alessandrino sconfisse gli AustroPiemontesi a Bassignana sul Tanaro, occupando le città di Asti, Casale e Tortona e assediando la grande Cittadella di Alessandria il 16 ottobre del 1745. La frontiera fortificata alessandrina vi avvierà ad un lungo periodo di stabilità fino al finire del secolo, quando il territorio e le sue fortezze vennero coinvolte nei fatti legati alla Rivoluzione Francese e all'epopea bonapartista.

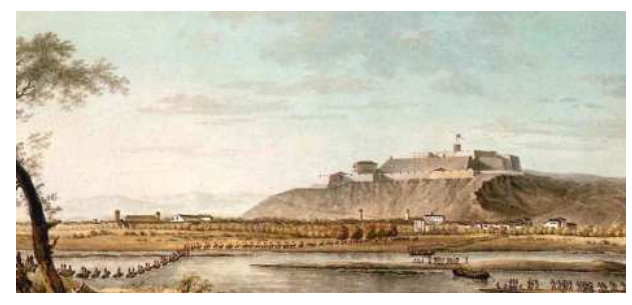

Fig. 4. Giuseppe Pietro Bagetti, Vue des villes et citadelles de Tortona... (3 mai 1796).

\section{La frontiera meridionale}

Come fu travagliata e tormentata la frontiera a nord ed est del territorio alessandrino, così fu relativamente calma, almeno fino alla fine del secolo XVIII quella sud, che vedeva nel forte di Gavi, (Figg. 3b e 5a) (Mandracci, Marotta 1994) posto lungo l'antica via Postumia che collegava Genova con il basso Piemonte e la Lombardia, il baluardo tra la Repubblica di Genova e i territori sabaudi. Frontiera relativamente calma in quanto la Repubblica di Genova, orbitante da sempre nell'area di interesse francese, non aveva mire espansionistiche verso nord, caso mani il contrario, erano i Savoia a guardare con avidità i porti liguri sul Mediterraneo. Il forte subì alcuni assedi, ma i più importanti fu quello del 1625 delle forze franco-savoiarde, perso e riconquistato dai genovesi in soli ventuno giorni e quello del 1746, durante la Guerra di Successione austria- 
ca, che assediato dagli austriaci capitolo su espressa sollecitazione della stessa Genova. Si fanno risalire agli anni Settanta del Seicento importanti lavori di ristrutturazione del forte di Gavi e del Monte Moro, coordinati dal Commissario generale della Repubblica Genovese Ansaldo de Mari. Verrà ampliata la capacità di alloggiare la truppa fino alla capacità di 900 fanti. Ma l'intervento di rifacimento più importante verrà attuato nel 1717 da Giovanni Gherardo de Langlade, ingegnere di grande esperienza formatosi nelle campagne delle Fiandre, della Polonia e del Portogallo. Il suo progetto di potenziamento del forte prevede l'aggiunta di false braghe e il rafforzamento dei bastioni del Maschio, oltre alla costruzione di un ampio edificio per il riparo delle truppe; l'ampliamento del rivellino verso la città, la presenza di due nuove casematte in corrispondenza del bastione di San Bernardo e della rampa di accesso coperto alla mezzaluna. Le costruzioni adibite a comando e alle truppe posti nel Maschio, sono rinforzate con spesse volte a "prova di bomba". Questi interventi permettono al forte di resistere all'assedio degli Austriaci del 1746, fino alla resa. Nel 1748 il forte di Gavi ritornerà alla Repubblica Genovese. La stessa frontiera meridionale ritornò strategica verso la fine del secolo XVIII, quando le truppe francesi provenienti dal sud della Francia, attraverso il territorio ligure, attaccarono gli Austro- Piemontesi in alcune note battaglie. Da sempre le inva- sioni galliche del territorio piemontese avevano come direttrici i passi alpini centrali e fino all'invasione francese per mezzo dell'Armata d'Italia agli ordini del generale Bonaparte, il confine sud del territorio alessandrino rimase inviolato. Però nella primavera del 1796 l'esercito rivoluzionario francese, proveniente dalla Liguria ingaggiò una battaglia con gli AustroPiemontesi prima a Dego (11 aprile 1796), poi a Montenotte (12 aprile) a Millesimo (13/14 aprile) e successivamente a Mondovì (20/21 aprile), per aprirsi la strada fino alla valle del Bormida e alla potente piazzaforte di Alessandria. Città e fortezza che nel giugno del 1800 caddero in mano napoleonica dopo la famosa battaglia di Marengo, che permise ai Francesi di impadronirsi di gran parte dell'Italia settentrionale.

Sulle frontiere alessandrine la "pax francese" dura cinquantanove anni. Nel 1859, durante la seconda Guerra d'Indipendenza, la fiamma della guerra si riaccese, come nei secoli passati, sulla frontiera est, confinate con il regno LombardoVeneto dominato dall'Austria, contro la quale combatte l'esercito franco-piemontese. Finalmente, questi furono gli ultimi fatti d'arme che interessarono la frontiera dell'alessandrino, che perse completamente la sua valenza strategica quando il Regno di Sardegna estese i suoi confini fino al lontano veneto, rimasto agli Austriaci fino al 1866 (Fig. 6).

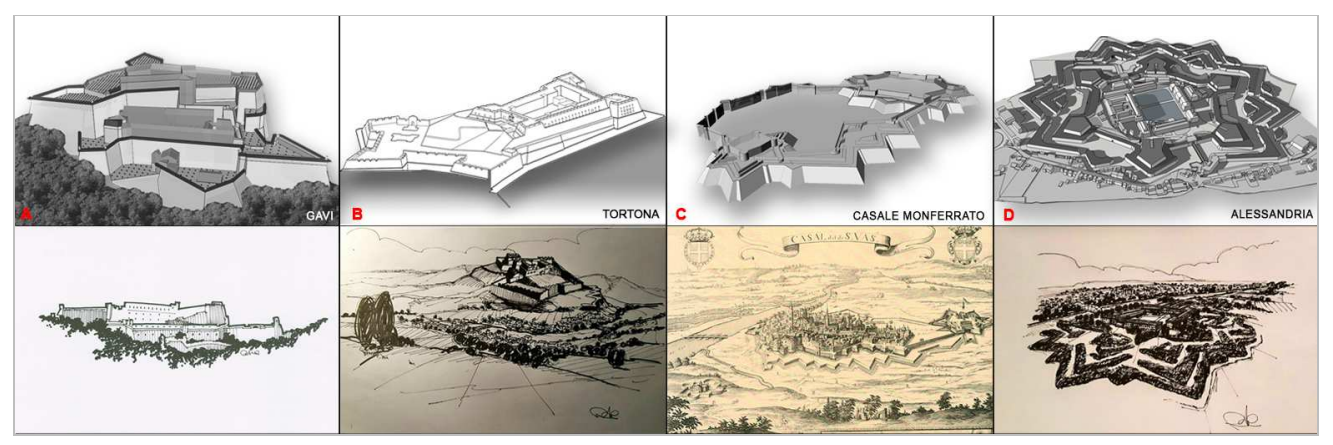

Fig. 5. Le fortificazioni del territorio alessandrino: a) Forte di Gavi (modellazione di V. Cirillo e schizzo di C. Rabino); b) Tortona e suo castello (modellazione di O. Zerlenga e schizzo di C. Rabino); c) Cittadella di Casale Monferrato (modellazione di V. Cirillo); d) Cittadella di Alessandria (modellazione di A. Marotta e schizzo di C. Rabino). Narrazione visiva dei forti con la modellazione tridimensionale, schizzi dal vero e iconografie storiche. 


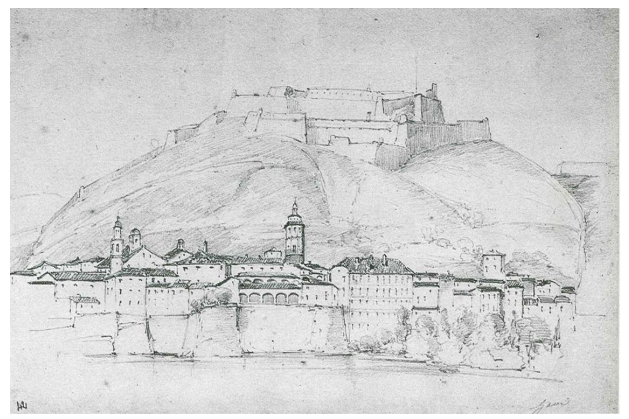

Fig. 6. Pasquale Domenico Cambiaso. Gavi. La rappresentazione di Gavi e del forte - tra "veduta" e "paesaggio" - dalla sponda opposta del fiume Lemme.

\section{Conclusioni}

Sia pure qui limitato in una sintesi storico-critica estremamente ridotta, il territorio della difesa nell'Alessandrino si conferma quale sistema connesso e integrato, inquadrato in un contesto internazionale, quale oggetto per una reale ed efficace valorizzazione (turistico-culturale, economica, paesistica e altro). La narrazione per immagini (dal più tradizionale disegno d'arte di Claudio Rabino alle più innovative renderizzazioni di Vincenzo Cirillo) bene evidenzia analogie e difformità nei caratteri essenziali delle singole fortezze, e nei relativi rapporti con il rispettivo territorio di pertinenza. Ma nello stesso tempo la medesima sintesi visiva vuole alludere alle potenzialità dell'intero sistema: ampliato, caratterizzato e articolato secondo il più complesso e specialistico concetto di "frontiera" (anche a seguito di recenti seminari internazionali, come quello di Almeida; CEAMA), potrà rivivere non più come apparato di difesa, ma come luogo (anche simbolico) di scambio di popoli, dalla guerra alla Pace, offrendosi come soggetto di nuove e più approfondite indagini, internazionali, interdisciplinari, interculturali.

\section{Bibliography}

Barghini, V.; Mandracci, C.; Marotta, A., eds. (1993). Valenza e le sue fortificazioni. Architettura e urbanistica dal Medioevo all'età contemporánea, SO.G. ED., Alessandria.

Bar-LeDuc, J.E. De. (1600). La Fortification reduicte en art et demonstrè̀, Libro III, Parigi.

Blengino, G.L. (1985). Viaggio nell'Italia dell'Ottocento dalle vedute di P. D. Cambiaso, Carige, Genova.

Cirillo, V.; Marotta, A.; Zerlenga, O. (2018). "Rappresentando il Forte di Gavi: ieri, oggi, domani”, in Marotta, A.; Spallone, R. eds., FORTMED 2018. Defensive architecture of the Mediterranean, Politecnico di Torino, Torino, vol. IX, pp. 1005-1012.

Forti, C.L. (1992). Fortificazioni e ingegneri militari in Liguria (1684-1814), Compagnia dei Librai, Genova.

Mandracci, V.C.; Marotta, A. (1995). Tortona e il suo castello. Dal dominio spagnolo al periodo postunitario, Cassa di Risparmio di Alessandria, Alessandria.

Mandracci, V.C.; Marotta, A., eds. (1994). Il Forte di Gavi in età moderna e contemporánea, SO.G. ED, Alessandria.

Marotta, A. (2013). "Cultura della visione per conservare e valorizzare: il caso della Cittadella di Alessandri”, in $35^{\circ}$ Convegno Internazionale dei Docenti della Rappresentazione. Patrimoni e Siti Unesco. Memoria, misura e armonia / Heritage and Unesco Sites. Memory, measure and harmony. Matera, 24-26 ottobre 2013, pp. 615-622.

Marotta, A. (2012). "Geometria e costruzione: modelli mentali e tipi realizzati nel territorio della difesa", DisegnareCon, 5, 9, pp. 161-166.

Marotta, A. ed. (1990a). La Cittadella di Alessandria. Una fortezza per il territorio dal Settecento all'unità, Cassa di Risparmio di Alessandria, Alessandria.

Marotta, A. ed. (1990b). La Cittadella di casale da fortezza del Monferrato a baluardo d'Italia 1590-1859, Cassa di Risparmio di Alessandria, Alessandria.

Migliari, R. (2003). Geometria dei modelli. Rappresentazione grafica e informatica per l'architettura e il design, Kappa, Roma.

N.A. (2019). "Soberania e fronteiras abaluartadas: da fronteira de Espanha no Piemonte (Alessandria, Itàlia) à Raia de Portugal. Programação do $13^{\circ}$ Seminàrio internacional de Almeida", CEAMA, 20.

Parodi, G. (1983). "Pietro Moretini, l'architetto della «ridotta di Montemoro»", in Fatti e profili di Gavi. 
Patrone, P.D.; Blengino, G.L. (1984). La Liguria di Ponente nell'Ottocento dalle vedute di P. D. Cambiaso, Ecig, Genova.

Perret de Chambery, J. (1601). Des Fortifications et artifices d'architecture et de perspective, Paris.

Quarenghi, C.; Gaspare Maculano o frate Vincenzo da Fiorenzuola Cardinale di S. Clemente ingegnere militare del Secolo XVII. Memorie storiche di Cesare Quarenghi tenente di fanteria e socio del R. Istituto d'Incoraggiamento di Napoli e dell' Ateneo di Brescia. ASCG, ms. 1079, s.d.

Raimondi, A. Di; Profumo, L.M. (1982). Bartolomeo Bianco a Genova, Genova.

Rubertis, R. de. (1994). Il disegno dell'architettura, La Nuova Italia Scientifica, Roma.

Viganò, M. (2014). Bibliografia della fortificazione - Parte generale e Stati italiani 1470-1945, Istituto Italiano dei Castelli, SEB Ed., Chiasso.

Zerlenga, O.; Cardone, V.; Cennamo, C. (2016). "Architetture della difesa a Nisida", in Verdiani, G. ed., FORTMED 2016. Defensive Architecture of the Mediterranean XV to XVIII Centuries. Atti del Congresso Internazionale Fortmed 2016 "Modern Age Fortifications of the Mediterranean Coast”, Firenze, vol. III, pp. 387-394.

Zerlenga, O. (1993). "Il disegno dell'architettura fortificata nel XVI secolo. Realtà costituite e fonti iconografiche a confronto, Tesi di Dottorato di Ricerca", in Rilievo e Rappresentazione del Costruito, VI Ciclo, Palermo. 